TITLE:

\title{
Experimental searches for antikaonic clusters
}

$\operatorname{AUTHOR}(\mathrm{S})$ :

Nagae, Tomofumi

\section{CITATION:}

Nagae, Tomofumi. Experimental searches for antikaonic clusters.

Nuclear Physics A 2012, 881: 141-149

ISSUE DATE:

2012-05

URL:

http://hdl.handle.net/2433/155797

\section{RIGHT:}

@ 2011 Elsevier B.V.; この論文は出版社版でありません。引用の際には 出版社版をご確認ご利用ください。; This is not the published version. Please cite only the published version. 


\title{
Experimental searches for antikaonic clusters ${ }^{\text {th }}$
}

\author{
Tomofumi Nagae \\ Department of Physics, Kyoto University, Kitashirakawa, Kyoto 606-8502, Japan
}

\begin{abstract}
The existence of antikaonic nuclear clusters, quasi-bound systems composed of an antikaon and a nucleus, is an important issue both theoretically and experimentally. By using all available experimental data, which are rather old and poor in statistics, a qualitative theoretical consensus is that antikaon nucleus interaction is attractive and strongly absorptive. However, there remain large uncertainties in quantitative estimates on these strengths, which prevents from drawing a definite conclusion. In the experimental side, new data are recently accumulated. Here I summarize the present experimental status on the antikaonic clusters mainly obtained in stopped $K^{-}$reactions.
\end{abstract}

Keywords: kaonic nuclei, $K^{-} p p$, multinucleon absorption

\section{Introduction}

The interactions of antikaon with nucleons and nuclei are a key issue to answer the question if antikaonic nuclear cluster exist or not. In the theoretical side, now we have a good frame work of chiral SU(3) effective field theory to describe the low-energy $\bar{K} N$ interactions. What about the experimental situation?

There are several old data in 1960's - 1970's on the $K^{-} p$ scattering at low energies [1] on $K^{-} p \rightarrow K^{-} p$ (elastic), $K^{-} p \rightarrow \bar{K}^{0} n$ (charge exchange), and $K^{-} p \rightarrow \pi^{0} \Lambda, \pi^{0} \Sigma^{0}, \pi^{ \pm} \Sigma^{\mp}$ (inelastic). There also exist three threshold branching ratios rather accurately measured; $\Gamma\left(K^{-} p \rightarrow \pi^{+} \Sigma^{-}\right) / \Gamma\left(K^{-} p \rightarrow \pi^{-} \Sigma^{+}\right)$, $\Gamma\left(K^{-} p \rightarrow \pi^{ \pm} \Sigma^{\mp}\right) / \Gamma\left(K^{-} p \rightarrow\right.$ all inelastic channels $)$, and $\Gamma\left(K^{-} p \rightarrow \pi^{0} \Lambda\right) /$

\footnotetext{
This work is partially supported by the Grant-In-Aid for Specially Promoted Research 23000003 by Japan Society for the Promotion of Science (JSPS).

Email address: nagae@scphys.kyoto-u.ac.jp (Tomofumi Nagae)
} 
$\Gamma\left(K^{-} p \rightarrow \pi^{0} \Lambda, \pi^{0} \Sigma^{0}\right)[2]$. The $K^{-} p$ scattering length is also deduced from the kaonic hydrogen X-ray measurements. The most recent SIDDHARTA data (Fig. 1) [3] has improved the accuracy compared with the previous ones reported from KEK-PS E228 [4] and the DEAR collaboration [5].

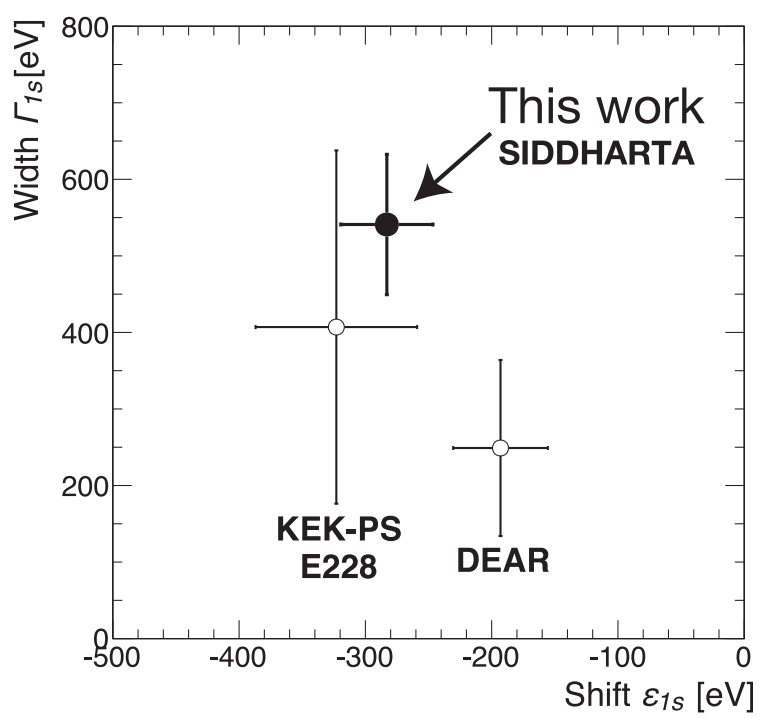

Figure 1: 1s-energy-level shifts and widths of kaonic hydrogen measurements due to strong interaction. Taken from Ref. [3].

The $K^{-}$-atom X-ray data in a wide mass-number range measured around 1970's are available to constrain the antikaon-nucleus interactions [6]. A reasonably good fit to the data is obtained with a density-dependent form of the phenomenological antikaon-nucleus potential. It suggests a rather deep $(\sim 160-200 \mathrm{MeV})$ real part of the potential. A large deviation observed in the ${ }^{4} \mathrm{He} 3 \mathrm{~d} \rightarrow 2 \mathrm{p}$ transition X-ray between a theoretical global fit and the old data has been solved in the recent measurements in KEK E570 [7] and in SIDDHARTA [8]. The SIDDHARTA collaboration has also reported a new measurement on ${ }^{3} \mathrm{He}[9]$.

All the theoretical investigations [10] using these available data suggest overall that there is a strong attraction in the antikaon-nucleus interactions. However, for the underlying $\bar{K} N$ interaction, there still remain considerable ambiguities arising from the $\bar{K} N$ sub-threshold region, where the $\Lambda(1405)$ plays an important role. 
With respect to the sub-threshold behavior of the antikaon nucleus interactions, the stopped $K^{-}$absorption reactions on various nuclei give us useful information. It has been well known [11] that there exist non-mesonic absorption modes at about $20 \%$ per stopped $K^{-}$in a wide range of nuclei from ${ }^{4} \mathrm{He}$ to heavy ones, while it is only about $1 \%$ for deuterium. In the case of ${ }^{4} \mathrm{He}$ [12], the non-mesonic modes amount to $16.4 \pm 2.6 \%$ and they are accompanied with $\Sigma^{+}(1.0 \pm 0.4 \%), \Sigma^{-}(3.6 \pm 0.9 \%), \Sigma^{0}(2.3 \pm 1 \%)$, and $\Lambda(9.4 \pm 2.6 \%)$. It should be noted that the effect of $\Sigma N \rightarrow \Lambda N$ conversion is not so significant for the non-mesonic absorption because of the high momentum $(\approx 500$ $\mathrm{MeV} / c)$ of the $\Sigma$ 's compared with the low momentum $\Sigma$ 's $(\approx 150 \mathrm{MeV} / c)$ emitted in the mesonic absorption processes $\left(K^{-} N \rightarrow \Sigma \pi\right)$.

\section{2. $\Lambda N$ pairs; $K^{-} p p$}

The first experimental evidence of a kaonic bound state, $K^{-} p p$, was reported from the FINUDA collaboration [13]. A lot of $\Lambda p$ pairs emitted in back-to-back in the lab. system were observed in the stopped $K^{-}$absorptions on ${ }^{6} \mathrm{Li},{ }^{7} \mathrm{Li}$, and ${ }^{12} \mathrm{C}$ nuclei. As mentioned in the previous section, it is not surprising to observe such events assuming they are coming from the non-mesonic absorption of $K^{-"} p p " \rightarrow \Lambda p$. However, the invariant mass distribution of the $\Lambda p$ pairs showed a large shift of about $115 \mathrm{MeV}$ toward the binding side (Fig. 2).

In the FINUDA experiment, higher statistics data were accumulated during the 2006-2007 data taking after the first data taking in 2003-2004. In the preliminary analyses of the data, it has been confirmed [14] that the invariant mass distribution of the $\Lambda p$ pairs emitted from the lightest target of ${ }^{6} \mathrm{Li}$ only has a similar shape to the first observation. A further analysis to understand the invariant mass distribution, opening angle distribution etc. is in progress considering various effects of final state interactions (FSI), $\Sigma N \rightarrow \Lambda N$ conversions, and so on. For example, it was suggested that the FSI effect on the proton and/or $\Lambda$ after the quasi free two-nucleon absorption would reduce the invariant mass of the $\Lambda p$ system [15], while the back-to-back correlation between the proton and $\Lambda$ would be lost. It seems difficult to explain both the invariant mass and opening angle distributions from such FSI effects.

Fujioka of the FINUDA collaboration also reported in the PANIC08 conference that such a large invariant mass shift is peculiar in the $\Lambda p$ pairs but not in the $\Lambda n$ pairs [16]. The $\Lambda n$ pairs, which are also emitted in back-to-back, show a major contribution coming from the quasi free two-nucleon absorp- 


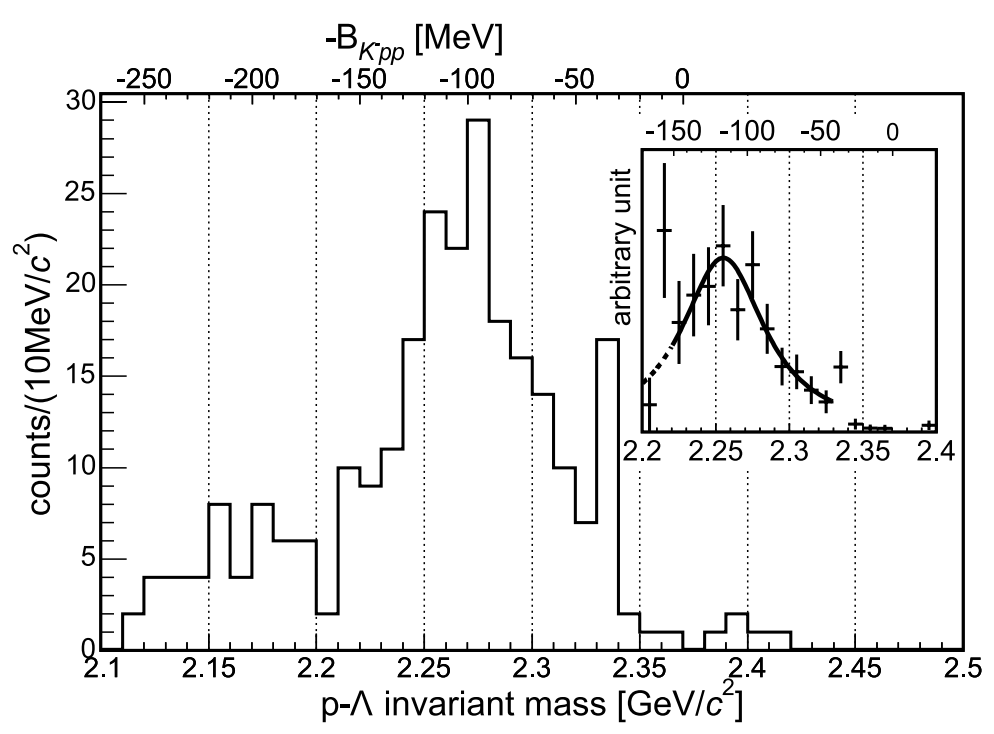

Figure 2: Invariant mass spectrum of the $\Lambda p$ pairs emitted in the stopped $K^{-}$absorption on ${ }^{6} \mathrm{Li},{ }^{7} \mathrm{Li}$, and ${ }^{12} \mathrm{C}$ targets measured in the FINUDA first data taking [13]. The inset shows the acceptance corrected spectrum with a fit curve.

tion process, $K^{-} " p n " \rightarrow \Lambda n$. A similar analysis of the stopped $K^{-}$reaction on ${ }^{4} \mathrm{He}$ in high statistics was reported from the KEK E549 experiment [17]. The invariant mass distributions of the $\Lambda p$ and $\Lambda n$ pairs were compared in a wide invariant-mass range from $2100 \mathrm{MeV} / c^{2}$ to $2400 \mathrm{MeV} / c^{2}$. It was found that the $\Lambda n$ pairs had a main component of about $2 \%$ per stopped $K^{-}$from the quasi free two-nucleon absorption and the $\Lambda p$ pairs had only about $0.2 \%$. It was also noticed a similar mass shift in the $\Lambda p$ pairs as observed in the FINUDA experiment. This large difference between the $\Lambda p$ and $\Lambda n$ pairs also seems very difficult to explain with FSIs only.

A similar binding of about $105 \mathrm{MeV}$ for the $K^{-} p p$ was also observed in an exclusive measurement in the $p+p \rightarrow K^{+}+\Lambda+p$ reaction at $2.85 \mathrm{GeV}$ by the DISTO group [18] (Fig. 3). A narrow signal at about $2200 \mathrm{MeV} / \mathrm{c}^{2}$ in the $\Lambda p$ invariant mass was reported by the OBELIX collaboration in the reaction of $\bar{p}^{4} \mathrm{He} \rightarrow\left(p \pi^{-}\right) p K_{s}^{0} X$ [19]. It might be attributed to a cusp effect at the threshold of $\Lambda p \pi$ [14].

Stimulated with these experimental results, several theory groups [20] carried out the calculations of the $K^{-} p p$ system based on few-body techniques. It is confirmed that the system has a bound state with the binding 


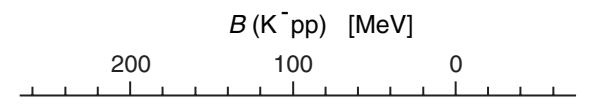

(a) large-angle proton: high $-P_{\mathrm{T}}(\mathrm{p})$

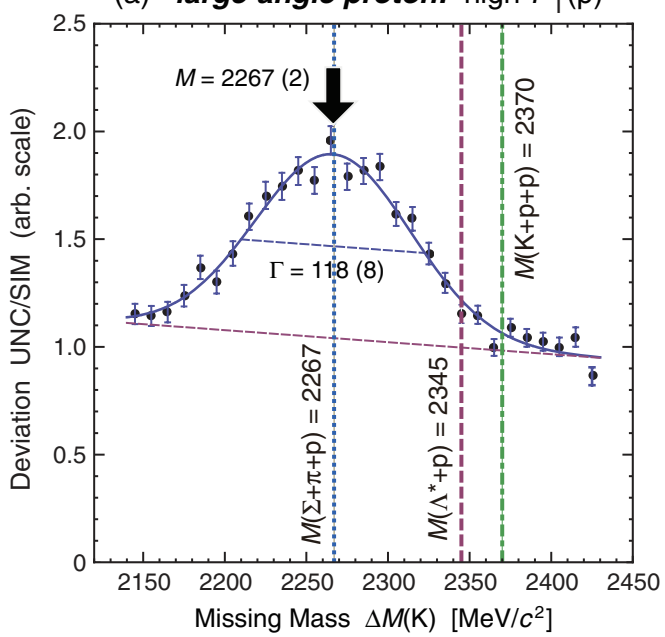

Figure 3: $K^{+}$missing mass spectrum divided by a simulated spectrum of the $p K^{+} \Lambda$ process in a uniform phase-space distribution obtained in the DISTO experiment [18]. A large-angle proton cut of $\left|\cos \theta_{c m}(p)\right|>0.6$ is applied.

energy of $40-90 \mathrm{MeV}$ and the broad width of $60-100 \mathrm{MeV}$ depending on their interaction models. Therefore, it is of vital importance to experimentally establish the existence of the lightest kaonic nucleus $K^{-} p p$ in the future experiments.

\section{3. $\Lambda d$ pairs}

It was a surprise that the FINUDA collaboration further obtained the back-to-back $\Lambda d$ pairs in coincidence in the stopped $K^{-}$reaction on ${ }^{6} \mathrm{Li}$ [21]. It is a good indication that a $K^{-}$is absorbed with three nucleons $p p n$. The invariant mass of the $\Lambda d$ pairs had a bump at around $3250 \mathrm{MeV} / c^{2}$ which is $\sim 60 \mathrm{MeV}$ below the $K^{-} p p n$ threshold. The estimated formation rate is $(4.4 \pm 1.4) \times 10^{-3}$ per stopped $K^{-}$in ${ }^{6} \mathrm{Li}$.

A better statistics data was also reported from the KEK E549 in the stopped $K^{-}$absorption on ${ }^{4} \mathrm{He}$ as shown in Fig. 4 [22]. The $\Lambda d n$ and $\Sigma^{0} d n$ components were separately identified. As suggested by a theoretical calculation [23], it seems that the quasi free three-nucleon absorption is dominant, 


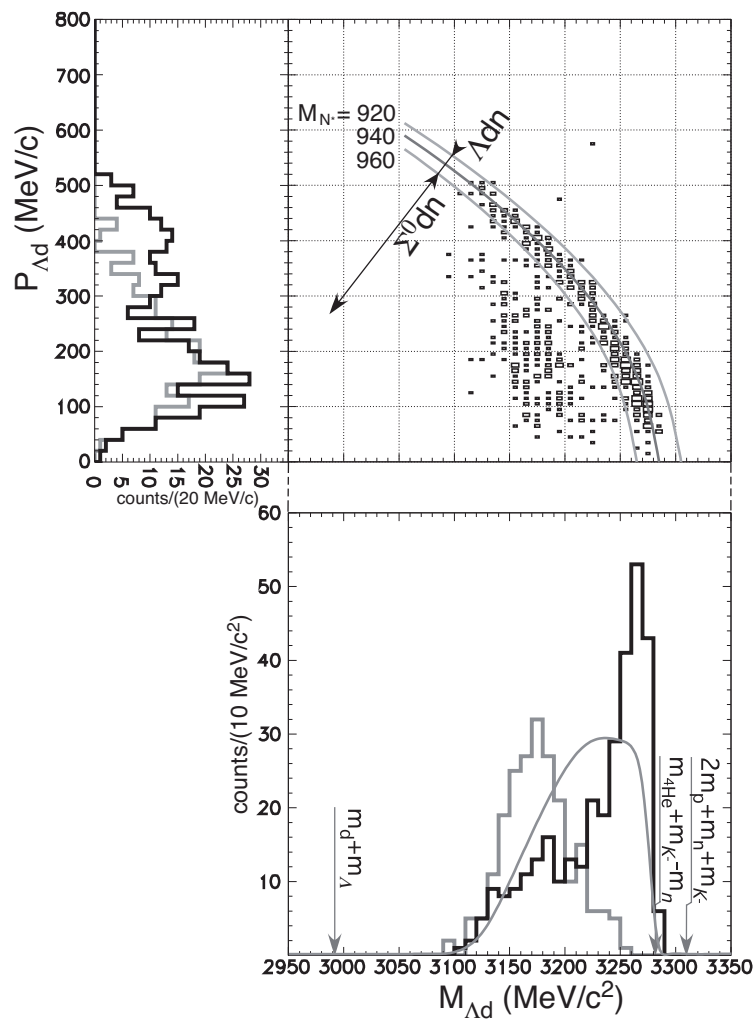

Figure 4: A correlation diagram of the invariant mass of $\Lambda d$ pairs $M_{\Lambda d}$ and the total momentum of the pair $P_{\Lambda d}$ measured in KEK E549 [22]. On the projections, contributions of the $\Lambda d n$ and $\Sigma^{0} d n$ events are represented by black and gray lines, respectively. The phase-space distribution is represented by a thin gray curve.

which should be also true for the FINUDA observation. The estimate of the formation rate was $(0.9 \pm 0.1$ (stat) \pm 0.2 (syst) $) \times 10^{-3}$ per stopped $K^{-}$in ${ }^{4} \mathrm{He}$.

\section{4. $\Lambda t$ pairs}

A further surprise came from the FINUDA experiment; back-to-back $\Lambda t$ pairs were detected as shown in Fig. 5 [24].

These events were cleanly identified as the back-to-back events (Fig. 6). Because of the limited number of events, we could not investigate the kinematical distributions so well. However, they seem to be consistent with $K_{\text {stop }}^{-} \mathrm{A} \rightarrow \Lambda(\gamma) t \mathrm{~A}^{\prime}$ and/or $K_{\text {stop }}^{-} \mathrm{A} \rightarrow \Lambda t N \mathrm{~A}^{\prime}$. The estimated absorption rate 


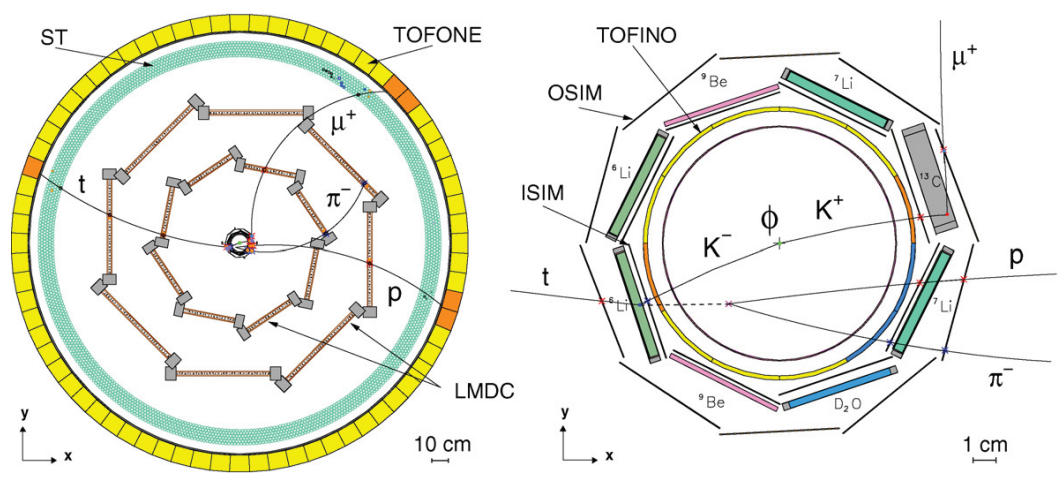

Figure 5: $\mathrm{A}{ }^{6} \operatorname{Li}\left(K_{\text {stop }}^{-}, \Lambda t\right) \mathrm{A}^{\prime}$ reconstructed event in the FINUDA experiment [24]. The right-hand side represents the inner region of the FINUDA spectrometer surrounding the $D A \Phi N E$ beam pipe, and the left-hand side depicts the outer tracker of FINUDA and TOFONE.

for the $K_{\text {stop }}^{-}{ }^{6} \mathrm{Li}$ is $(7.1 \pm 3.4) \times 10^{-4}$ per stopped $K^{-}$. It should be noted that it is the same level as that for the three-nucleon absorption of $10^{-3}$.

The similar analysis was also done in KEK E549 [25]. The back-to-back $\Lambda t$ pairs were observed with a branching ratio of $(3.3 \pm 0.4$ (stat) \pm 0.5 (syst) $) \times$ $10^{-4}$ which is consistent with the previous value of $(3 \pm 2) \times 10^{-4} / K_{\text {stop }}^{-}[26]$.

\section{Discussion}

Summarizing the experimental observations in the FINUDA experiment and KEK E549, the quasi free two-nucleon absorption was identified by detecting the $\Lambda N$ pairs emitted in back-to-back for the first time, although such non-mesonic absorption mode has been known to exist at the rate of $\sim 20 \%$ per stopped $K^{-}$. However, the quasi free two-nucleon absorption was only dominant in the $\Lambda n$ mode at about $2 \%$ per stopped $K^{-}$and not in the $\Lambda p$ mode (only $0.2 \% / K_{\text {stop }}^{-}$). Non quasi free parts are dominant for the $\Lambda p$ mode, and it can be a signal of the $K^{-} p p$ bound state. In addition to the two-nucleon absorption, three-nucleon absorption of $\sim 10^{-3}$ into the $\Lambda d$ pairs, and four-nucleon absorption of more than $1 / 3$ of the three-nucleon absorption into the $\Lambda t$ pairs were observed in the stopped $K^{-}$absorption.

Naively thinking, these numbers should be too large. Suppose we have three-nucleon absorption, we expect the dominant decay mode would be $Y N N$ emission into three-body phase space because of a large Q-value. The 


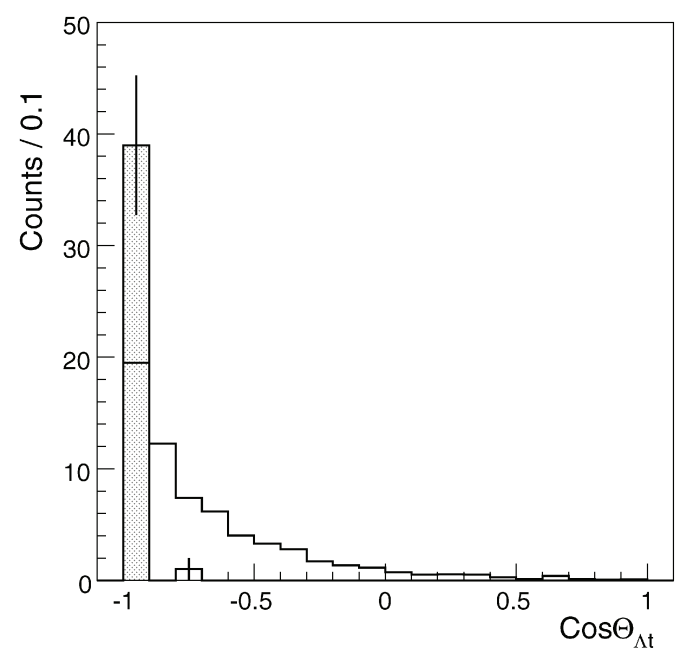

Figure 6: Opening angle distribution between $\Lambda$ and $t$ pairs $\left(\cos \Theta_{\Lambda t}\right)$ from Ref. [24]. Filled histogram, experimental data; open histogram, phase space simulation for the $K_{\text {stop }}^{-} A \rightarrow$ $\Lambda t N A^{\prime}$ reaction.

$\Lambda d$ final state has a very limited phase space. We should also have a $\Sigma$ in the final state as well as a $\Lambda$. This means the total branching fraction of threenucleon absorption could be an order of magnitude larger $\left(O\left(10^{-2}\right)\right)$ than the observed rate of the $\Lambda d$ mode. The same argument should be true for the four-nucleon absorption observed in the $\Lambda t$ mode, which could even suggest the four-nucleon absorption might take place more than the three-nucleon absorption.

If this is not the case, we should have some reasons. Otherwise, we, experimentalists, were too lucky to observe such complicated multi-nucleon absorption processes in the easiest detectable modes of $\Lambda+d$ or $t$ emitted in back-to-back in which the detector only covered such high-momentum $d$ and $t$. The $\Lambda$ decay mode looks dominant compared with the $\Sigma^{ \pm}$modes for which the detectors need to measure neutral particles from the $\Sigma^{ \pm}$decay.

Here, I would like to refer to a recent theoretical paper by T. Sekihara, D. Jido, and Y. Kanada-En'yo [27] on the non-mesonic decay of kaonic nuclei. In their analysis, they investigated the non-mesonic decay under a $\Lambda(1405)$ doorway picture. Namely, they estimated the decay branching ratios of $\Lambda(1405) N \rightarrow \Lambda N$ and $\Sigma N$ with one-meson exchange. They found the $\Gamma_{\Lambda N} / \Gamma_{\Sigma^{0} N}$ ratio was strongly dependent on the coupling ratios of $\Lambda(1405)$ 
with $\bar{K} N\left(G_{\bar{K} N}\right)$ and with $\pi \Sigma\left(G_{\pi \Sigma}\right)$ in nuclear medium as shown in Fig. 7 .

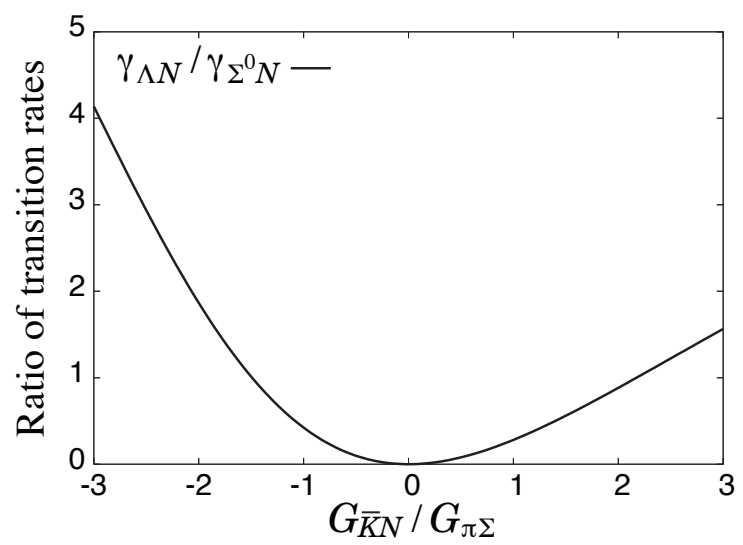

Figure 7: Ratio of the transition rates of $\Lambda^{*} N$ to $\Lambda N$ and $\Sigma^{0} N, \gamma_{\Lambda N} / \gamma_{\Sigma^{0} N}$, as a function of $G_{\bar{K} N} / G_{\pi \Sigma}$ calculated in Ref. [27].

In their calculation, the coupling ratio of $G_{\bar{K} N} / G_{\pi \Sigma}$ was taken from the chiral unitary approach value of 2.5 . It leads to the $\Gamma_{\Lambda N} / \Gamma_{\Sigma^{0} N}$ value of $\approx 1.2$. Taking account of the other $\Sigma^{ \pm}$branches, they obtained the $\Sigma$ dominance over the $\Lambda N$ mode.

Here I should note that the experimental $\Gamma_{\Lambda N} / \Gamma_{\Sigma^{0} N}$ ratio for ${ }^{4} \mathrm{He}$ is $\approx 4$ as mentioned in section 1 , not $\approx 1.2$. It suggests that the $G_{\bar{K} N} / G_{\pi \Sigma}$ value is around -3 from Fig. 7 . If this is the case, the $\Lambda$ mode is dominant than the $\Sigma$ mode. In addition, when the $\Lambda(1405)$ couples to $\pi \Sigma$, the nucleons in the final state are produced with the virtual pion absorption by two or more nucleons (Fig. 8(a)), through which the nucleons involved should be energetically emitted separately, not as a deuteron nor a triton cluster. On the other hand, if the $\Lambda(1405)$ strongly couples to $\bar{K} N$ in medium, a virtual $\bar{K}$ propagates among the multi-nucleons involved, as shown in Fig. 8(b), by exciting each nucleon in to a $\Lambda(1405)$. The excited $\Lambda(1405)$ also couples to $\bar{K} N$, and produces a nucleon in low-energy because of the small $Q$ value. As the result, in the final state, a nucleon cluster could be easily formed from the low-energy nucleons and a $\Lambda$ is additionally emitted. 
(a)

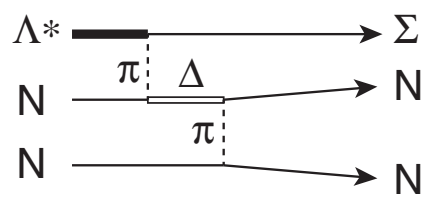

(b)

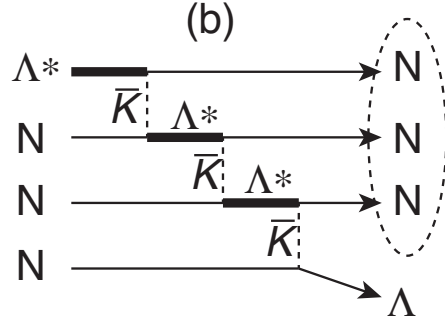

Figure 8: Possible diagrams contributing to multi-nucleon absorption reactions through the $\Lambda(1405) N$ as a doorway; (a) in the $\pi \Sigma$ mode, and (b) in the $\bar{K} N$ mode.

\section{Summary}

In the stopped $K^{-}$absorption reactions, non-mesonic absorption rate has been known to be about $20 \%$ per stopped $K^{-}$. The quasi free two-nucleon absorption process is experimentally identified in the $\Lambda n$ pairs emitted in back-to-back, which amounts to about $2 \% / K_{\text {stop }}^{-}$. On the other hand, in the $\Lambda p$ mode, the quasi free component is only about $0.2 \% / K_{\text {stop }}^{-}$and there exists a bump structure attributable to the $K^{-} p p$ bound state. The future experiments to confirm the existence of antikaon clusters are strongly awaited.

The quasi free three-nucleon absorption (four-nucleon absorption) is identified with the $\Lambda d(\Lambda t)$ pairs emitted in back-to-back with high momentum. The branching fraction of the order of $0.1 \%(\gtrsim 0.03 \%)$ is observed in such a specific two-body decay mode. A strong $\Lambda(1405)$ coupling to the $\bar{K} N$ rather than the $\pi \Sigma$ in nuclear medium might have a possibility to explain the experimental situation above.

\section{References}

[1] References included in the following theoretical analyses,

A.D.Martin, Nucl. Phys. B 179 (1981) 33-48,

N. Kaiser, P.B. Siegel, W. Weise, Nucl. Phys. A 594 (1995) 325-345.

[2] D.N. Tovee et al., Nucl. Phys. B 33 (1971) 493-504, R.J. Nowak et al., Nucl. Phys. B 139 (1978) 61-71.

[3] M. Bazzi et al., Phys. Lett. B 704 (2011) 113-117. 
[4] M. Iwasaki et al., Phys. Rev. Lett. 78 (1997) 3067-3069; T. Ito et al., Phys. Rev. C 58 (1998) 2366-2382.

[5] G. Beer et al., Phys. Rev. Lett. 94 (2005) 212302.

[6] C.J. Batty, E. Friedman, A. Gal, Phys. Rep. 287 (1997) 385-445.

[7] S. Okada et al., Phys. Lett. B 653 (2007) 387-391.

[8] M. Bazzi et al., Phys. Lett. B 681 (2009) 310-314.

[9] M. Bazzi et al., Phys. Lett. B 697 (2011) 199-202.

[10] E. Friedman and A. Gal, Phys. Rep. 452 (2007) 89-153, W. Weise, Nucl. Phys. A 835 (2010) 51-58.

[11] C. Vander Velde-Wilquest et al., Il Nuovo Cimento 39 A (1977) 538-547.

[12] P.A. Katz et al., Phys. Rev. D 1 (1970) 1267-1276.

[13] M. Agnello et al., Phys. Rev. Lett. 94 (2005) 212303.

[14] S. Piano, Nucl. Phys. A 835 (2010) 35-42.

[15] V.K. Magas, E. Oset, A. Ramos, and H. Toki, Phys. Rev. C 74 (2006) 025206 .

[16] H. Fujioka, in: I. Tserruya, A. Gal, and D. Ashery (Eds.), Proc. of the eighteenth Particles and Nuclei International Conference-PANIC08, Eilat, Israel, 9-14 November, 2008, Elsevier, pp.441-443.

[17] T. Suzuki et al., Mod. Phys. Lett. A 23 (2008) 2520-2523.

[18] T. Yamazaki et al., Phys. Rev. Lett. 104 (2010) 132502.

[19] G. Bendiscioli et al., Eur. Phys. J. A 40 (2009) 11-22.

[20] N.V. Shevchenko, A. Gal, J. Mareš and J. Révai, Phys. Rev. C 76 (2007) 044004.

Y. Ikeda and T. Sato, Phys. Rev. C 79 (2009) 035201.

T. Yamazaki and Y. Akaishi, Phys. Rev. C 76 (2007) 045201.

A. Doté, T. Hyodo, and W. Weise, Phys. Rev. C 79 (2009) 014003. 
[21] M. Agnello et al., Phys. Lett. B 654 (2007) 80-86.

[22] T. Suzuki et al., Phys. Rev. C 76 (2007) 068202.

[23] V.K. Magas, E. Oset, and A. Ramos, Phys. Rev. C 77 (2008) 065210.

[24] M. Agnello et al., Phys. Lett. B 669 (2008) 229-234.

[25] T. Suzuki et al., arXiv:[nucl-ex]1009.5082v1.

[26] M. Roosen and J.H. Wickens, Nuovo Cimento 66 A (1981) 101-113.

[27] T. Sekihara, D. Jido, Y. Kanada-En'yo, Phys. Rev. C 79 (2009) 062201. 